\title{
Composition, taxonomy and functional diversity of the oropharynx microbiome in individuals with schizophrenia and controls
}

Eduardo Castro-Nallar, Matthew L Bendall, Marcos Pérez-Losada, Sarven Sabuncyan, Emily G. Severance, Faith B. Dickerson, Jennifer R. Schroeder, Robert H. Yolken, Keith A. Crandall

The role of the human microbiome in schizophrenia remains largely unexplored. The microbiome has been shown to alter brain development and modulate behavior and cognition in animals through gut-brain connections, and research in humans suggests that it may be a modulating factor in many disorders. This study reports findings from a shotgun metagenomic analysis of the oropharyngeal microbiome in 16 individuals with schizophrenia and 16 controls. High-level differences were evident at both the phylum and genus levels, with Proteobacteria, Firmicutes, Bacteroidetes, and Actinobacteria dominating both schizophrenia patients and controls, and Ascomycota being more abundant in schizophrenia patients than controls. Controls were richer in species but less even in their distributions, i.e., dominated by fewer species, as opposed to schizophrenia patients. Lactic acid bacteria were relatively more abundant in schizophrenia, including species of Lactobacilli and Bifidobacterium, which have been shown to modulate chronic inflammation. We also found Eubacterium halii, a lactate-utilizing species. Functionally, the microbiome of schizophrenia patients was characterized by an increased number of metabolic pathways related to metabolite transport systems including siderophores, glutamate, and vitamin B12. In contrast, carbohydrate and lipid pathways and energy metabolism were abundant in controls. These findings suggest that the oropharyngeal microbiome in individuals with schizophrenia is significantly different compared to controls, and that particular microbial species and metabolic pathways differentiate both groups. Confirmation of these findings in larger and more diverse samples, e.g., gut microbiome, will contribute to elucidating potential links between schizophrenia and the human microbiota. 
1 Eduardo Castro-Nallar ${ }^{1}$, 6* ${ }^{*}$ Matthew L. Bendall ${ }^{1}$, Marcos Pérez-Losada ${ }^{1,}$ 5, 7, Sarven

2 Sabuncyan², Emily Severance², Faith Dickerson³, Jennifer R. Schroeder ${ }^{4}$, Robert Yolken²,

3 and Keith A. Crandall ${ }^{1}$

4

$5 \quad{ }^{1}$ Computational Biology Institute, George Washington University, Ashburn, VA 20147

$6 \quad$ 2Stanley Neurovirology Laboratory, Johns Hopkins School of Medicine, Baltimore, MD

7 33heppard Pratt Hospital, Baltimore, MD

$8 \quad{ }^{4}$ Schroeder Statistical Consulting LLC, Ellicott City MD

$9{ }^{5}$ CIBIO-InBIO, Centro de Investigação em Biodiversidade e Recursos Genéticos,

10 Universidade do Porto, Vairão 4485-661

$11{ }^{6}$ Center for Bioinformatics and Integrative Biology, Universidad Andrés Bello, Facultad de

12 Ciencias Biológicas, Santiago, Chile

$13{ }^{7}$ Division of Emergency Medicine, Children's National Medical Center, Washington, DC

14 20010, USA

15 *Corresponding author: castronallar@gmail.com; 45085 University Dr., Innovation Hall

16 suite 305, Ashburn, VA 20147; Office: (571) 553-0221, Cell: (801) 318-9220

17 


\section{Introduction}

Schizophrenia is a serious neuropsychiatric disorder with substantial social and

21 economic consequences such as low performance on social knowledge and emotion

22 recognition, high family burden, and high annual treatment costs worldwide, especially in

23 treatment-resistant subjects (Achim et al. 2013; Kennedy et al. 2014; Knapp et al. 2004).

24 Population-based studies suggest that the risk of an individual developing schizophrenia is

25 based on both genetic and environmental factors (van Os et al. 2010); e.g., concordance-

26 rates between monozygotic twins are higher than those between dizygotic twins, and

27 adopted children of schizophrenic parents have similar risks of schizophrenia to those of

28 their biological parents (Fowler et al. 2012; Tienari et al. 1985). Additionally, a recent

29 genome-wide association study examining a large cohort of subjects $(36,989$ cases and

30113,075 controls) found 108 independent associated loci that appeared to account for $7 \%$

31 of a given person's risk of developing schizophrenia, as assessed by polygenic risk scores.

32 Moreover, some of the key loci were related to host immunity, thus providing genetic

33 support for immune dysregulation in schizophrenia (Schizophrenia Working Group of the

34 Psychiatric Genomics Consortium 2014). Likewise, a population-based study has identified

35 that single-nucleotide polymorphisms associated with schizophrenia are also associated

36 with immunity (Andreassen et al. 2014), which has also been confirmed in

37 clinical/epidemiological studies (Benros et al. 2014; Eaton et al. 2010). More studies

38 linking immunity/immune disorders and schizophrenia have been published (reviewed in

39 Severance et al. 2013), however the source of the immune activation in most individuals

40 has not been identified. 
42 the study of unculturable/difficult to culture microbial communities and have shown that

43 the human body harbors microbial ecosystems that interact with human physiological

44 processes, thus influencing health and disease (Cho \& Blaser 2012; de Vos \& de Vos 2012;

45 Pflughoeft \& Versalovic 2012). The collection of genes and genomes belonging to these

46 microbial communities, the microbiome, has been studied using HTS to demonstrate the

47 extent to which the human microbiota plays a role in human health issues ranging from 48 obesity to respiratory disease (Huang 2013; Turnbaugh et al. 2006).

In relation to human health, observational and experimental studies have shown that the microbiome can modulate the immune response and its alterations could be associated with disease (reviewed in Round \& Mazmanian 2009). For instance, a recent experimental study using an osteomyelitis mice model has shown that diet can modulate the microbiome (high fat diet induces a decrease in Prevotella), which in turn offered protection against inflammatory bone disease (Lukens et al. 2014). Increasing evidence suggests that alteration of the microbiota not only has effects on intestinal conditions such as Crohn's and other Inflammatory Bowel Diseases (Gevers et al. 2014; Morgan et al. 2012),

57 but also in the development of systemic immune diseases such as rheumatoid arthritis (Scher \& Abramson 2011; Wu et al. 2010), type I diabetes (Kriegel et al. 2011; Suez et al. 2014), and allergic diseases (Castro-Nallar et al. 2015; Hong et al. 2010; Nakayama et al. 2011; Pérez-Losada et al. 2015).

61 Although the mechanisms by which microorganisms outside the brain might impact 62 the central nervous system (CNS) are not known, the human microbiota have been 63 demonstrated to affect brain development and to modulate cognition through imbalances 
64 in the microbiota-gut-CNS axis (reviewed in Clarke et al. 2012; Davari et al. 2013; Foster \&

65 McVey Neufeld 2013; Hsiao et al. 2013). As a consequence, microbiota alterations impact

66 anxiety-like and depression-like behaviors and have been linked to neurodevelopmental

67 disorders such as autism spectrum disorder (Kang et al. 2013) and multiple sclerosis

68 (Farrokhi et al. 2013; Yokote et al. 2008).

While there is growing evidence suggesting a key role for the human microbiome in

mental health, the microbiome of schizophrenia patients has not yet been extensively

explored (reviewed in Dinan et al. 2014; Severance et al. 2014; Yolken \& Dickerson 2014).

Recent work by our group has shown that the oropharyngeal "phageome" of individuals with schizophrenia differs from that of non-schizophrenic controls. In particular, when controlling for age, gender, race, socioeconomic status, or cigarette smoking (Yolken et al. 2015). However, studies evaluating the structure and diversity of bacterial and fungal communities in schizophrenia individuals are not available. In particular, gut and oral microbiome analysis could provide insights into the identity and quantity of the microbes residing in these body sites, and whether they encode functions that are relevant to and/or known to be involved in schizophrenia. In addition, the analysis of the oral microbiome could prove instrumental in the development of taxonomic and functional biomarkers

82 because of the ease of sampling, which can be performed in a non-traumatic manner,

83 allowing to distinguish schizophrenia patients from healthy individuals, as it has been done 84 in other illnesses (e.g., Farrokhi et al. 2013).

The premise of our study is that the oropharyngeal microbiome may be associated 86 with or contribute to an altered immune state consistent with findings in schizophrenia; 
87 hence, differences in the microbiome could be instrumental to pinpoint associations

88 between microbial diversity and immune response. Consequently, here we aim to

89 characterize the schizophrenia microbiome by interrogating the oropharyngeal

90 microbiome structure regarding its taxonomic and functional diversity. This represents a

91 second crucial step towards understanding the relationship between microbiome diversity

92 and schizophrenia. Our initial study identified differences in the oropharyngeal phageome;

93 whereas here we focus on the complete microbiome (virus, bacteria, fungi). Future work

94 will investigate additional microbiome compartmentalizations, including gut, and amniotic

95 fluid. 


\section{Materials and Methods}

98 Samples and Sequencing

99 Participants were individuals with schizophrenia and non-psychiatric controls from

100 the Stanley Research Program at Sheppard Pratt Hospital who were enrolled in the period

101 between January 1, 2008 and March 1, 2012 in a study of the association between

102 antibodies to infectious agents and serious mental illness. The methods for identification

103 and recruitment of individuals with schizophrenia and controls have been previously

104 described (Dickerson et al. 2013). Briefly, individuals with schizophrenia were recruited

105 from psychiatric treatment programs at a large psychiatric health system and community

106 psychiatric programs in central Maryland. Inclusion criteria were: a diagnosis of

107 Schizophrenia or Schizoaffective disorders (established by consensus of the research team

108 based on the SCID for DSM-IV Axis 1 Disorders - Patient Edition and available medical

109 records). Participants met the following additional criteria: age 18-65; proficient in English;

110 absence of any history of intravenous substance abuse; absence of mental retardation;

111 absence of HIV infection; absence of serious medical disorder that would affect cognitive

112 functioning; absence of a primary diagnosis of alcohol or substance use disorder (First et al.

113 2012). In order to define an individual with schizophrenia, we used the criteria from the

114 DSM IV manual of the American Psychiatric Association (American Psychiatric Association

115 2000). The Institutional Review Boards of Sheppard Pratt Hospital and the Johns Hopkins

116 School of Medicine approved this study. Informed consent was obtained from all

117 participants prior to enrollment into the study (Protocols SMRI/SPHS: 2002-01 and 118 SF/SPHS: 2000-02). 
We selected the oropharynx microbiome since it is easily accessible by non-invasive

120 techniques and biological samples from cases and controls could be collected and

121 processed in an identical manner. Moreover, studies have found the oral microbiome to be

122 associated with immune and neurological diseases such as inflammatory bowel disease and

123 Alzheimer's disease, and offers opportunities for development of taxonomic and functional

124 biomarkers (Docktor et al. 2012; Farrokhi et al. 2013; Shoemark \& Allen 2014).

125 Throat swabs were obtained at the study visit (16 individuals with schizophrenia

126 and 16 controls) by research staff that rubbed a sterile cotton swab at the back of subjects'

127 throat and then immediately put the swab into a sterile container. The swabs were either

128 sent immediately to the processing laboratory or refrigerated and then sent to the

129 processing laboratory in a refrigerated container. Throat swabs were kept frozen at -70

130 degrees Celsius until further processing. DNA was extracted from throat swabs using

131 Qiagen's Gentra Puregene Buccal Cell Kit. The collection brush heads from the swab ends

132 were excised and incubated at $65^{\circ} \mathrm{C}$ overnight in the kit cell lysis solution. Aliquots of $75-$

$133100 \mathrm{ng}$ of DNA were used to generate sequencing libraries using the Nugen Ultralow DR

134 Multiplex System following the manufacturer's instructions. Briefly, following sonication,

135 the DNA fragments were end repaired and ligated to barcoded adaptors. Using the adaptors

136 as PCR priming sites, the library was amplified using 15 cycles of amplification. The

137 samples were purified by chromatography and analyzed by capillary electrophoresis in a

138 Bioanalyzer 2100 to confirm size and concentration. Sequencing libraries were matched

139 one case and one control per lane and anonymized until analysis. Samples were sequenced

140 at the same time to further minimize batch effects. Sequences were generated using the

141 Illumina HiSeq 2000 platform producing approximately 58-million single-end reads of 100 
142 nucleotides in length per sample (Suppl. File 1). All sequence data were deposited in the

143 Sequence Read Archive and are available under the BioProject PRJNA255439.

144 Sequence Read Preprocessing

145 Reads were preprocessed using PRINSEQ-lite 0.20.4 (trimming reads and bases $<25$

146 PHRED, removing exact duplicates, and reads with undetermined bases). We constructed a

147 'target' genome library containing all bacterial, fungal, and viral sequences from the Human

148 Microbiome Project $\quad$ Reference Database

149 (http://www.hmpdacc.org/reference_genomes/reference_genomes.php; 131 archeal, 326

150 lower eukaryotes, 3683 viral, 1751 bacterial species) using the PathoLib module from

151 PathoScope 2.0 (Hong et al. 2014). We aligned the reads to these libraries using the

152 Bowtie2 algorithm (Langmead \& Salzberg 2012), and then filtered any reads that also

153 aligned to the human genome (hg19) as implemented in PathoMap (--very-sensitive-local -

154 k 100 --score-min L,20,1.0). We then applied PathoScope 2.0 - specifically the PathoID

155 module (Francis et al. 2013) - to obtain accurate read counts for downstream analysis.

156 Statistical Analyses

157 Exploratory and differential species abundance analyses were performed in R 3.1.2

158 and Bioconductor 3.0 (Gentleman et al. 2004) using packages xlsx 0.5.7, gtools 3.4.1,

159 CHNOSZ 1.0.3.1, plyr 1.8.1, ggplot2 1.0.0, reshape2 1.4.1, gplots 2.16.0, Phyloseq 1.10.0, and

160 DESeq2 1.6.3 (Love et al. 2014; McMurdie \& Holmes 2013; Wickham 2009). Briefly, various

161 indices (Richness: Observed, Chao1, ACE; richness and evenness: Shannon, Simpson,

162 Inverse Simpson, Fisher's alpha) were obtained using the plot_richness function of the

163 PhyloSeq package and beta diversity was obtained using R base package. We used richness

164 indices to estimate the number of species in the microbiome with (Chao1; ACE) (Chao 
165 1984; Colwell \& Coddington 1994) and without (Observed) correction for subsampling. In

166 addition, we used metrics that aim to measure diversity by accounting for "evenness" or

167 homogeneity (Shannon; Fisher; Simpson; InvSimpson) (Jost 2007). For instance, 168 communities dominated by one species will exhibit low evenness, as opposed to 169 communities where species are relatively well represented (high evenness).

170 For statistical comparison between cases and controls, the number of mapped reads

171 estimated in PathoScope was normalized across all samples using the variance stabilized 172 transformation method as implemented in DESeq2 using a generalized linear model (Love 173 et al. 2014). Then, statistical inference was performed using the negative binomial Wald 174 test [with Cook's distance to control for outliers (Cook 1977)] and adjusted by applying the 175 Benjamini-Hochberg method to correct for multiple hypotheses testing (Benjamini \& 176 Hochberg 1995) at an alpha value $=0.01$. We controlled for covariates by adding them as 177 coefficients in DESeq's linear model.

178 We also used generalized linear models implemented in STAMP and MaAsLin 179 (Morgan et al. 2012; Parks et al. 2014) to test for differences between groups. In STAMP, 180 differences in relative abundance between two groups of samples were compared using

181 White's non-parametric $t$ test (White et al. 2009). We estimated confidence intervals using 182 a percentile bootstrapping method (10,000 replications), and false discovery rate (FDR) in 183 multiple testing was controlled by the Storey's FDR at 0.05 (Storey et al. 2004). MaAsLin is 184 a multivariate statistical framework that finds associations between clinical metadata and 185 microbial community abundance. These associations are without the influence of the other 186 metadata in the study. In our study, we used MaAsLin to detect the effect of schizophrenia 187 (presence/absence) in microbiome species composition taking into account the effects of 
188 other variables (confounders) in the study population (medication, smoker, age, gender 189 and race).

190 Descriptive statistics were run on all samples. Cases and controls were compared 191 with respect to demographic and substance use variables; $\chi^{\square}$ tests were used for 192 categorical variables and $t$ tests were used for continuous variables. Principal coordinate 193 analysis (PCoA) was performed on a Jensen-Shannon distance matrix derived from read 194 counts aggregated by genus as estimated in PathoScope.

195 In order to explore and formally test for differences in the coding potential of the 196 oropharyngeal microbiome, non-human reads were mapped against the Kyoto 197 Encyclopedia of Genes and Genomes (Kanehisa \& Goto 2000) (KEGG; from June 2011; 1981291309 genes with KO assignments) database using a two-stage local alignment 199 algorithm as implemented in UBLAST (e-value $1 \mathrm{e}^{-9}$ ), part of the USEARCH package 200 v7.0.1090 (Edgar 2010). Then, metabolic pathway abundance and coverage was estimated 201 using the Human Microbiome Project metabolic reconstruction pipeline, HUMAnN v0.99, 202 where pathways are inferred as gene sets using maximum parsimony as the optimality 203 criterion [MinPath (Ye \& Doak 2009)] and smoothed-averaged over all genes within a 204 pathway. Significant differences between groups were tested using Kruskal-Wallis rank 205 sum and Wilcoxon tests (alpha $=0.05$ ) using Linear Discriminant Analysis as implemented 206 in LEfSe (Segata et al. 2011). All figures were plotted using the ggplot2 and PhyloSeq 207 packages.

\section{Results}

209 Study sample demographic variables 
The study sample consisted of 16 schizophrenia patients and 16 controls.

212 Participants had a mean age of 34.5 years, were $56.3 \%$ male, and $37.5 \%$ white. On average,

213 their mothers had over 13 years of education, and 31.3\% of participants smoked. Cases

214 were more likely to be cigarette smokers than controls $\left(\chi^{2}=18.6 ; p\right.$ value $<0.0001 ; 62.5 \%$

215 and $0 \%$, respectively) and were also more likely to have a higher body mass index (BMI;

216 controls $=25.5$, cases $=34.7 ; p$ value $<0.0001)$. Groups did not differ significantly on

217 demographic variables such as maternal education, self-reported race, age, or gender

218 (Table 1).

219 Microbial communities in the oropharynx of schizophrenia patients differ significantly from 220 those in controls

221 At the phylum level, schizophrenia samples exhibit higher proportions of Firmicutes

222 across samples in comparison to controls, where we observe higher relative proportions of 223 Bacteroidetes and Actinobacteria (Fig. 1). Relative proportions of other phyla such as

224 Fusobacteria and Proteobacteria do not differ greatly (Fig. 1). Overall, groups do not differ 225 significantly at the phylum level, which is also supported by non-metric multidimensional 226 scaling (Suppl. Fig. 1B; NMDS; Bray-Curtis dissimilarity). Differences between smoker and 227 non-smoker cases are not evident at the phylum level (Fig. 1; smoker cases denoted with a 228 star).

229 Regarding species diversity among samples, we observe that controls are richer in 230 the number of species compared to schizophrenia samples. The median number of 231 observed species is higher than the interquartile range of schizophrenia samples, which is 232 congruent with other richness metrics (Chao1, ACE, and Fisher; Suppl. Fig. 1A), suggesting 233 that controls contain a higher number of lower abundance species as opposed to 
234 schizophrenia samples. However, we observe that species abundance in controls is 235 dominated by fewer species (Streptococcus spp.; Fig. 1), as evidenced by their lower 236 evenness or homogeneity (Simpson, and Inverse Simpson; Suppl. Fig. 1A), although both 237 groups are fairly equivalent in species richness when accounting for species evenness 238 (Shannon; Suppl. Fig. 1A). Chao1 index provides an estimate of the expected number of 239 species in a habitat. We found that the observed richness is similar to Chao1 richness, 240 suggesting that we are capturing most of the diversity present in the samples (Suppl. Fig. 241 1A).

242 Microbial species commonly inhabiting the oropharynx are differentially more abundant in 243 schizophrenia patients than in controls

244 Out of a total of 25 differentially abundant species (bacteria and fungi), we found 6 245 microbial species to be more abundant in cases than controls after accounting for different 246 library sizes, smoking condition and medication (covariates were added as extra 247 coefficients; Wald test; $p$ value $<0.01$ ). Overall, schizophrenia samples were relatively more 248 abundant for Lactic Acid Bacteria (LAB) including Lactobacillus and Bifidobacterium. Of 249 these, the largest effect was observed in Lactobacillus gasseri, which appeared to be at least 250400 times more abundant in schizophrenia patients than controls $\left(\log _{2}=8.4 \pm 1.2\right.$ standard 251 error). We also detected Eubacterium halii, a lactate-utilizing bacterium present in human 252 feces, and Candida dubliniensis, which is an opportunistic fungus that is part of the oral 253 fungal microbiome (Table 2).

254 Using STAMP, we also found Streptococcus gordonii, Streptococcus thermophilus, and 255 Streptococcus $s p$. (oral taxon 071) to be relatively more abundant in schizophrenia. The 
256 latter species was also detected by MaAsLin, which in addition detected Bifidobacterium 257 pseudocatenulatum, Bifidobacterium breve (Suppl. File 3).

258 We also identified species that were related to variables other than 259 schizophrenia/non-psychiatric controls. Among these, we found that some species were 260 related to individuals' age (Neisseria subflava, Neisseria flavescens, Neisseria polysaccharea, 261 Escherichia fergusonii, and Pseudomonas protegens), being white (Klebsiella variicola, 262 Actinomyces phage_Av-1, Streptococcus sp. [oral taxon 071]), and to cigarette smoking 263 (Streptococcus mitis, Streptococcus pneumoniae) (Suppl. File 3). Regarding species 264 relatively more abundant in cases, we found that these preferentially belonged to 265 Pasteurella, Neisseriaceae, and Flavobacteriaceae. We also collated the list of species found 266 in schizophrenia patients against a list of potential contaminants published by Salter et al. 267 and found no obvious contaminants (Table 2; Salter et al. 2014).

We also tested whether microbial composition could differentiate schizophrenia 269 patients from controls by inferring synthetic variables that could explain the variability of 270 the samples at the genus level (PCoA on Jensen-Shannon distance; Fig. 2). We observe that 271 schizophrenia samples tend to group together (first three coordinates $=40 \%, 25 \%$ and $27223 \%$ of variance, respectively), however, this differentiation seems to be influenced by 273 mental health status and in part by cigarette smoking (Fig. 2A and B).

\section{Microbial metabolic pathways differ between schizophrenics and controls}

276 value $<0.05$; Fig. 3). Pathways significantly associated with schizophrenia were related to 277 environmental information processing, in particular to transport systems such as 278 saccharide, polyol, and lipid transport systems (M00197, M00194, M00200), peptide and 
279 nickel transport (M00239), metallic cation, iron-siderophore, and vitamin B12 transport 280 (M00246), and phosphate and amino acid transport (M00222), including glutamate 281 transport (M00233). In turn, pathways found in control individuals, but not in individuals 282 with schizophrenia, were related to energy metabolism, such as ATP synthesis and ATP 283 synthase (M00144, M00150, M00157, M00164) and carbohydrate and lipid metabolism, 284 such as central carbohydrate metabolism (M00011, M00009, M00007) and 285 lipopolysaccharide metabolism (M00060). 


\section{Discussion}

This study is among the first surveys on the composition and differences in the microbiome of schizophrenia patients and healthy controls using shotgun metagenomic sequencing. The oropharyngeal microbiome is particularly attractive for microbiomeassociated biomarker development because biological samples can be collected and processed in an identical and non-traumatic manner from both individuals with psychiatric disorders and controls. Additionally, while oral and gut microbiomes share little taxonomic resemblance, both are significantly associated (Ding \& Schloss 2014), which might be instrumental for future diagnostic aids. In addition, shifts in oral microbiome diversity have been linked to immune and neurological diseases such as inflammatory bowel disease and Alzheimer's disease (Docktor et al. 2012; Shoemark \& Allen 2014). Although there is little mechanistic understanding about the role of the microbiome on inflammatory and neurological disease, some studies have found specific markers (microbes and metabolites) associated with both gut and oral microbiomes. These markers have been shown to enter the systemic circulation and elicit systemic immune responses, thus serving as specific biomarkers of disease (Clark et al. 2013; Farrokhi et al. 2013).

We show that the oropharynx microbiome in schizophrenics is significantly different in comparison from that of healthy controls. High-level differences were evident at both the phylum and genus levels; however, the overall composition of the microbiome is similar to those previously reported (Belda-Ferre et al. 2011; Dewhirst et al. 2010). Proteobacteria, Firmicutes, Bacteroidetes, and Actinobacteria dominated both schizophrenia patients and controls, with Ascomycota being more abundant in schizophrenia patients than controls. At the genus level, we identified key members of the 
311 healthy oropharynx microbiome such as Prevotella, Capnocytophaga, Campylobacter,

312 Veillonella, Streptococcus, Neisseria and Haemophilus; as reported in other studies that

313 characterize the oropharynx microbiome (Charlson et al. 2010). We also detected in

314 schizophrenia samples an increase in lactic acid bacteria, Candida, and Eubacterium, and a

315 marked reduction of Neisseria, Haemophilus, and Capnocytophaga, suggesting a

316 characteristic dysbiosis signature (Gao et al. 2014). Notably, decreases in Neisseria and

317 Capnocytophaga abundance have been associated with cigarette smoking (Charlson et al.

318 2010); therefore, although we incorporate cigarette smoking as a coefficient in our models,

319 we can not rule out that this observation is due to smoking and not mental health status.

320 We also found that Streptococcus mitis and Streptococcus pneumoniae abundance was

321 associated with smoking, which is consistent with other findings from culture independent

322 (Charlson et al. 2010) and culture-based studies (Brook \& Gober 2005).

323 Reagent and laboratory contamination in genomics in general (Merchant et al. 2014;

324 Strong et al. 2014) and in microbiome studies in particular (Lusk 2014; Salter et al. 2014;

325 Weiss et al. 2014) have been recently brought into attention as an alarming problem.

326 Although contamination affects primarily samples coming from low diversity ecosystems,

327 in our analysis we used high statistical significance $(p$ value $<0.01$ coupled with

328 independent filtering and outlier removal) to prevent spurious detection of contaminants

329 as differentially abundant. Moreover, our laboratory protocols (library preparations;

330 sequencing) were performed simultaneously for all samples, and both case and control

331 samples were loaded in the same sequencing lane, thus minimizing chances of

332 contamination, i.e., contamination correlated with disease status. 
Some of the most abundant species found in samples from individuals with

334 schizophrenia (e.g., Lactobacillus, Bifidobacterium, Candida) have been reported previously

335 as members of the respiratory tract microbiome and have been linked to opportunistic

336 infections (Kim \& Sudbery 2011; Land et al. 2005). However, relatively abundant species in

337 schizophrenia samples are not exclusive inhabitants of the respiratory tract, as bacterial

338 species we detected have also been described in the gastrointestinal tract (Dillon 1998;

339 Duncan et al. 2004; Kageyama \& Benno 2000). In addition, some reports show that oral and

340 intestinal Lactobacilli share a common origin in the oral cavity (Dal Bello \& Hertel 2006),

341 suggesting that species such as L. salivarius and L. gasseri (both identified in this study)

342 might be allochthonous to the human gut and autochthonous to the respiratory tract

343 (Reuter 2001), and therefore potentially useful for microbiome signature development

344 (Aagaard et al. 2012; Haberman et al. 2014). In addition, Lactobacilli (except L. salivarius)

345 in the intestine has been linked to emotional behavior regulation and alteration of mRNA

346 expression patterns of the GABA receptor via the vagus nerve (Bravo et al. 2011).

347 Lactobacilli have also been linked to chronic inflammation and anxiety-like behavior

348 (Bercik et al. 2010). In particular, L. gasseri has been shown to modulate the immune

349 system by altering the function of dendritic cells, enterocytes, and components of innate

350 immunity (Luongo et al. 2013; Selle \& Klaenhammer 2013).

351 We also detected non-bacterial species such as fungi and DNA viruses (phage; Suppl.

352 File 2) that would have been beyond the scope of gene marker surveys, e.g., 16S rRNA gene

353 (Weinstock 2012). One fungal species, Candida dubliniensis, was differentially abundant in

354 individuals with schizophrenia, which has been associated to immune-compromised

355 individuals (Eggimann \& Pittet 2014; Sebti et al. 2001), but also found in healthy 
356 respiratory tract microbiomes (prevalence of 75\% Candida) (Ghannoum et al. 2010). This

357 suggests that the high abundance of these microbes in schizophrenia individuals might be

358 associated with altered immune responses or changes in the local environment that enable

359 their outgrowth, as observed in other diseases (Bisgaard et al. 2007; Molyneaux et al.

360 2013). We also detected viruses, primarily phages, in our datasets; however our group has

361 published a more comprehensive characterization of them in a larger study population (see

362 Yolken et al. 2015).

363 We also assessed the functional diversity of the oropharynx microbiome in

364 schizophrenia and control samples. There is little information regarding the functional

365 diversity of the respiratory tract in general, and a complete lack of information in

366 schizophrenia. In our study, we identified metabolic pathways significantly associated with

367 the microbiomes of schizophrenics (i.e., environmental information processing) including

368 glutamate transport (M00233), while the microbiomes of healthy controls were enriched

369 for bioenergy pathways. Microbial metabolic pathways found in schizophrenia samples

370 have also been described in samples from the respiratory and digestive tracts such as in

371 gingiva, oral cavity, and stool (M00239, M00276, M00200, M0028, M0029), reinforcing the

372 notion that oral and gut microbial communities share features and are associated (Meehan

373 \& Beiko 2012; Sczesnak et al. 2011; Seekatz et al. 2014; Segata et al. 2012). Dominant

374 hypotheses for the pathophysiology of schizophrenia, as well as recent genetic studies,

375 point to neurotransmitter disturbances in glutamatergic and dopaminergic activities

376 (Falkai et al. 2011; Schizophrenia Working Group of the Psychiatric Genomics Consortium

377 2014). While we found glutamate related pathways to be more abundant in schizophrenia

378 samples, the design and scope of this study does not allow us to evaluate mechanistic 
379 hypotheses regarding pathophysiology of schizophrenia and the potential contribution of 380 the microbiome. Study designs based on metatransciptomics or dual RNAseq strategies 381 coupled with advanced statistical techniques such as feature reduction might be 382 instrumental to detect associations between known host genetic determinants and their 383 expression, and microbiota structure (Morgan et al. 2015). Undoubtedly, study designs 384 using larger sample sizes and both RNA and DNA information collected from different body 385 sites (e.g., intestines) are needed for accurate statistical validation of microbial and 386 metabolic as promising biomarkers for schizophrenia. Further analyses of other body sites 387 involving larger study populations will be presented elsewhere as we collect relevant 388 samples.

389 In summary, by using metagenomic sequencing, we have shown that it is possible to 390 distinguish schizophrenia patients from controls by profiling the oropharyngeal

391 microbiome based on the diversity and composition of microbes. Additionally, 392 microbiomes from schizophrenics and controls differ in the functions they potentially 393 encode suggesting it may be important to further characterize other body sites such as the 394 intestines. These differences could be exploited for the development of biomarkers and 395 ultimately for therapeutic interventions.

396 The fact that all controls were non-smokers, although statistically accounted for in 397 our inferences, might confound the effects of schizophrenia from those of smoking on 398 microbiome composition. A recent study found no significant differences in diversity (alpha 399 and beta) between the oral microbiome in healthy smokers and non-smokers without 400 psychiatric disorders (Morris et al. 2013). However, this and other studies have found 401 specific taxa (Haemophilus influenzae, Streptococcus pneumoniae, Megasphaera and 
402 Veillonella spp) to be differentially abundant in smokers and non-smokers (in humans

403 Charlson et al. 2010; in mice Voss et al. 2015). Here we found that the distributions of two

404 Streptococcus species were better explained by whether the individual was a smoker than

405 whether the individual had schizophrenia.

406 Additionally, studies in animal models have indicated that changes in the

407 microbiome can cause alterations in behavior and cognitive functioning and that these

408 changes can be modulated by probiotic and antibiotic interventions (Jakobsson et al. 2010;

409 Martin et al. 2008). The establishment of a link between the microbiome and behavioral

410 and cognitive functioning in humans might lead to the development of new strategies for

411 the prevention, management, and treatment of psychiatric disorders.

412

413 Acknowledgements

414

415

We thank The Stanley Medical Research Institute for their support of this work. We

416 also thank the GWU Colonial One High Performance Computing Cluster for computational

417 time.

418 
442

443

444

445

446

447

448

449

450

451

452

453

454

455

456

457

458

459

460

461

462

463

464

465

\section{References}

Aagaard K, Riehle K, Ma J, Segata N, Mistretta T-A, Coarfa C, Raza S, Rosenbaum S, Van den Veyver I, and Milosavljevic A. 2012. A metagenomic approach to characterization of the vaginal microbiome signature in pregnancy. PloS one 7:e36466.

Achim AM, Ouellet R, Lavoie M-A, Vallières C, Jackson PL, and Roy M-A. 2013. Impact of social anxiety on social cognition and functioning in patients with recent-onset schizophrenia spectrum disorders. Schizophrenia research 145:75-81.

American Psychiatric Association. 2000. Diagnostic and statistical manual of mental disorders, text revision (DSM-IV-TR): American Psychiatric Association.

Andreassen O, Harbo H, Wang Y, Thompson W, Schork A, Mattingsdal M, Zuber V, Bettella F, Ripke S, and Kelsoe J. 2014. Genetic pleiotropy between multiple sclerosis and schizophrenia but not bipolar disorder: differential involvement of immune-related gene loci. Molecular Psychiatry 10:207-214.

Belda-Ferre P, Alcaraz LD, Cabrera-Rubio R, Romero H, Simón-Soro A, Pignatelli M, and Mira A. 2011. The oral metagenome in health and disease. The ISME journal 6:46-56.

Benjamini Y, and Hochberg Y. 1995. Controlling the false discovery rate: a practical and powerful approach to multiple testing. Journal of the Royal Statistical Society Series B (Methodological) 57:289-300.

Benros ME, Nielsen PR, Nordentoft M, Eaton WW, Dalton SO, and Mortensen PB. 2014. Autoimmune diseases and severe infections as risk factors for schizophrenia: a 30year population-based register study. American Journal of Psychiatry 168:13031310.

Bercik P, Verdu EF, Foster JA, Macri J, Potter M, Huang X, Malinowski P, Jackson W, Blennerhassett $P$, and Neufeld KA. 2010. Chronic gastrointestinal inflammation induces anxiety-like behavior and alters central nervous system biochemistry in mice. Gastroenterology 139:2102-2112. e2101.

Bisgaard H, Hermansen MN, Buchvald F, Loland L, Halkjaer LB, Bønnelykke K, Brasholt M, Heltberg A, Vissing NH, and Thorsen SV. 2007. Childhood asthma after bacterial colonization of the airway in neonates. New England Journal of Medicine 357:14871495.

Bravo JA, Forsythe P, Chew MV, Escaravage E, Savignac HM, Dinan TG, Bienenstock J, and Cryan JF. 2011. Ingestion of Lactobacillus strain regulates emotional behavior and central GABA receptor expression in a mouse via the vagus nerve. Proceedings of the National Academy of Sciences 108:16050-16055.

Brook I, and Gober AE. 2005. Recovery of potential pathogens and interfering bacteria in the nasopharynx of smokers and nonsmokers. CHEST Journal 127:2072-2075.

Castro-Nallar E, Shen Y, Freishtat RJ, Pérez-Losada M, Manimaran S, Liu G, Johnson WE, and Crandall KA. 2015. Integrating Metagenomics and Host Gene Expression to Characterize Asthma-Associated Microbial Communities. BMC Medical Genomics in press.

Chao A. 1984. Nonparametric estimation of the number of classes in a population. Scandinavian Journal of statistics 11: 265-270.

Charlson ES, Chen J, Custers-Allen R, Bittinger K, Li H, Sinha R, Hwang J, Bushman FD, and Collman RG. 2010. Disordered microbial communities in the upper respiratory tract of cigarette smokers. PloS one 5:e15216. 
466

Cho I, and Blaser MJ. 2012. The human microbiome: at the interface of health and disease. Nature Reviews Genetics 13:260-270.

Clark RB, Cervantes JL, Maciejewski MW, Farrokhi V, Nemati R, Yao X, Anstadt E, Fujiwara M, Wright KT, and Riddle C. 2013. Serine lipids of Porphyromonas gingivalis are human and mouse Toll-like receptor 2 ligands. Infection and immunity 81:34793489.

Clarke G, Grenham S, Scully P, Fitzgerald P, Moloney R, Shanahan F, Dinan T, and Cryan J. 2012. The microbiome-gut-brain axis during early life regulates the hippocampal serotonergic system in a sex-dependent manner. Molecular Psychiatry 18:666-673.

Colwell RK, and Coddington JA. 1994. Estimating terrestrial biodiversity through extrapolation. Philosophical Transactions of the Royal Society of London Series B: Biological Sciences 345:101-118.

Cook RD. 1977. Detection of influential observation in linear regression. Technometrics 19:15-18.

Dal Bello F, and Hertel C. 2006. Oral cavity as natural reservoir for intestinal lactobacilli. Systematic and applied microbiology 29:69-76.

Davari S, Talaei S, and Alaei H. 2013. Probiotics treatment improves diabetes-induced impairment of synaptic activity and cognitive function: Behavioral and electrophysiological proofs for microbiome-gut-brain axis. Neuroscience 240:287296.

de Vos WM, and de Vos EA. 2012. Role of the intestinal microbiome in health and disease: from correlation to causation. Nutrition reviews 70:S45-S56.

Dewhirst FE, Chen T, Izard J, Paster BJ, Tanner AC, Yu W-H, Lakshmanan A, and Wade WG. 2010. The human oral microbiome. Journal of bacteriology 192:5002-5017.

Dickerson F, Stallings C, Origoni A, Vaughan C, Khushalani S, Yang S, and Yolken R. 2013. Creactive protein is elevated in schizophrenia. Schizophrenia research 143:198-202.

Dillon VM. 1998. Lactic acid Bacteria: Microbiology and Functional Aspects. International Journal of Food Science \& Technology 33:195-196.

Dinan T, Borre Y, and Cryan J. 2014. Genomics of schizophrenia: time to consider the gut microbiome? Molecular Psychiatry 19:1252-1257.

Ding T, and Schloss PD. 2014. Dynamics and associations of microbial community types across the human body. Nature 509:357-360.

Docktor MJ, Paster BJ, Abramowicz S, Ingram J, Wang YE, Correll M, Jiang H, Cotton SL, Kokaras AS, and Bousvaros A. 2012. Alterations in diversity of the oral microbiome in pediatric inflammatory bowel disease. Inflammatory bowel diseases 18:935-942.

Duncan SH, Louis P, and Flint HJ. 2004. Lactate-utilizing bacteria, isolated from human feces, that produce butyrate as a major fermentation product. Applied and environmental microbiology 70:5810-5817.

Eaton WW, Pedersen MG, Nielsen PR, and Mortensen PB. 2010. Autoimmune diseases, bipolar disorder, and non - affective psychosis. Bipolar disorders 12:638-646.

Edgar RC. 2010. Search and clustering orders of magnitude faster than BLAST. Bioinformatics 26:2460-2461.

Eggimann P, and Pittet D. 2014. Candida colonization index and subsequent infection in critically ill surgical patients: 20 years later. Intensive care medicine 40:1429-1448. 
510 Falkai P, Schmitt A, and Cannon TD. 2011. Pathophysiology of schizophrenia. Chichester, UK. $511 \quad$ : John Wiley \& Sons, Ltd.

512 Farrokhi V, Nemati R, Nichols FC, Yao X, Anstadt E, Fujiwara M, Grady J, Wakefield D, Castro W, and Donaldson J. 2013. Bacterial lipodipeptide, Lipid 654, is a microbiomeassociated biomarker for multiple sclerosis. Clinical \& Translational Immunology 2:e8.

First MB, Spitzer RL, Gibbon M, and Williams JB. 2012. Structured Clinical Interview for DSM-IV® Axis I Disorders (SCID-I), Clinician Version, Administration Booklet: American Psychiatric Pub.

Foster JA, and McVey Neufeld K-A. 2013. Gut-brain axis: how the microbiome influences anxiety and depression. Trends in neurosciences 36:305-312.

Fowler T, Zammit S, Owen MJ, and Rasmussen F. 2012. A population-based study of shared genetic variation between premorbid IQ and psychosis among male twin pairs and sibling pairs from Sweden. Archives of general psychiatry 69:460-466.

Francis OE, Bendall M, Manimaran S, Hong C, Clement NL, Castro-Nallar E, Snell Q, Schaalje GB, Clement MJ, and Crandall KA. 2013. Pathoscope: Species identification and strain attribution with unassembled sequencing data. Genome research 23:1721-1729.

Franzosa EA, Morgan XC, Segata N, Waldron L, Reyes J, Earl AM, Giannoukos G, Boylan MR, Ciulla D, and Gevers D. 2014. Relating the metatranscriptome and metagenome of the human gut. Proceedings of the National Academy of Sciences 111:E2329-E2338.

Gao Z, Kang Y, Yu J, and Ren L. 2014. Human Pharyngeal Microbiome May Play A Protective Role in Respiratory Tract Infections. Genomics, proteomics \& bioinformatics 12:144150.

Gentleman RC, Carey VJ, Bates DM, Bolstad B, Dettling M, Dudoit S, Ellis B, Gautier L, Ge Y, and Gentry J. 2004. Bioconductor: open software development for computational biology and bioinformatics. Genome biology 5:R80.

Gevers D, Kugathasan S, Denson LA, Vázquez-Baeza Y, Van Treuren W, Ren B, Schwager E, Knights D, Song SJ, and Yassour M. 2014. The treatment-naive microbiome in newonset Crohn's disease. Cell host \& microbe 15:382-392.

Ghannoum MA, Jurevic RJ, Mukherjee PK, Cui F, Sikaroodi M, Naqvi A, and Gillevet PM. 2010. Characterization of the oral fungal microbiome (mycobiome) in healthy individuals. PLoS pathogens 6:e1000713.

Haberman Y, Tickle TL, Dexheimer PJ, Kim M-O, Tang D, Karns R, Baldassano RN, Noe JD, Rosh J, and Markowitz J. 2014. Pediatric Crohn disease patients exhibit specific ileal transcriptome and microbiome signature. The Journal of clinical investigation 124:3617.

Hong C, Manimaran S, Shen Y, Perez-Rogers JF, Byrd AL, Castro-Nallar E, Crandall KA, and Johnson WE. 2014. PathoScope 2.0: a complete computational framework for strain identification in environmental or clinical sequencing samples. Microbiome 2:1-15.

Hong P-Y, Lee BW, Aw M, Shek LPC, Yap GC, Chua KY, and Liu W-T. 2010. Comparative analysis of fecal microbiota in infants with and without eczema. PloS one 5:e9964.

Hsiao EY, McBride SW, Hsien S, Sharon G, Hyde ER, McCue T, Codelli JA, Chow J, Reisman SE, and Petrosino JF. 2013. Microbiota modulate behavioral and physiological abnormalities associated with neurodevelopmental disorders. Cell 155:1451-1463.

Huang YJ. 2013. Asthma microbiome studies and the potential for new therapeutic strategies. Current allergy and asthma reports 13:453-461. 
Jakobsson HE, Jernberg C, Andersson AF, Sjölund-Karlsson M, Jansson JK, and Engstrand L. 2010. Short-term antibiotic treatment has differing long-term impacts on the human throat and gut microbiome. PloS one 5:e9836.

Jost L. 2007. Partitioning diversity into independent alpha and beta components. Ecology 88:2427-2439.

Kageyama A, and Benno Y. 2000. Catenibacterium mitsuokai gen. nov., sp. nov., a grampositive anaerobic bacterium isolated from human faeces. International journal of systematic and evolutionary microbiology 50:1595-1599.

Kanehisa M, and Goto S. 2000. KEGG: kyoto encyclopedia of genes and genomes. Nucleic acids research 28:27-30.

Kang D-W, Park JG, Ilhan ZE, Wallstrom G, LaBaer J, Adams JB, and Krajmalnik-Brown R. 2013. Reduced incidence of Prevotella and other fermenters in intestinal microflora of autistic children. PloS one 8:e68322.

Kennedy JL, Altar CA, Taylor DL, Degtiar I, and Hornberger JC. 2014. The social and economic burden of treatment-resistant schizophrenia: a systematic literature review. International clinical psychopharmacology 29:63-76.

Kim J, and Sudbery P. 2011. Candida albicans, a major human fungal pathogen. The Journal of Microbiology 49:171-177.

Knapp M, Mangalore R, and Simon J. 2004. The global costs of schizophrenia. Schizophrenia bulletin 30:279.

Kriegel MA, Sefik E, Hill JA, Wu H-J, Benoist C, and Mathis D. 2011. Naturally transmitted segmented filamentous bacteria segregate with diabetes protection in nonobese diabetic mice. Proceedings of the National Academy of Sciences 108:11548-11553.

Land MH, Rouster-Stevens K, Woods CR, Cannon ML, Cnota J, and Shetty AK. 2005. Lactobacillus sepsis associated with probiotic therapy. Pediatrics 115:178-181.

Langmead B, and Salzberg SL. 2012. Fast gapped-read alignment with Bowtie 2. Nature methods 9:357-359.

Love MI, Huber W, and Anders S. 2014. Moderated estimation of fold change and dispersion for RNA-Seq data with DESeq2. Genome Biol 15:550.

Lukens JR, Gurung P, Vogel P, Johnson GR, Carter RA, McGoldrick DJ, Bandi SR, Calabrese CR, Walle LV, and Lamkanfi M. 2014. Dietary modulation of the microbiome affects autoinflammatory disease. Nature 516:246-249.

Luongo D, Miyamoto J, Bergamo P, Nazzaro F, Baruzzi F, Sashihara T, Tanabe S, and Rossi M. 2013. Differential modulation of innate immunity in vitro by probiotic strains of Lactobacillus gasseri. BMC microbiology 13:298.

Lusk RW. 2014. Diverse and widespread contamination evident in the unmapped depths of high throughput sequencing data. PloS one 9:e110808.

Martin FPJ, Wang Y, Sprenger N, Yap IK, Lundstedt T, Lek P, Rezzi S, Ramadan Z, van Bladeren P, and Fay LB. 2008. Probiotic modulation of symbiotic gut microbial-host metabolic interactions in a humanized microbiome mouse model. Molecular systems biology 4:157.

McMurdie PJ, and Holmes S. 2013. phyloseq: an R package for reproducible interactive analysis and graphics of microbiome census data. PloS one 8:e61217.

Meehan CJ, and Beiko RG. 2012. Lateral gene transfer of an ABC transporter complex between major constituents of the human gut microbiome. BMC microbiology $12: 248$. 
602 Merchant S, Wood DE, and Salzberg SL. 2014. Unexpected cross-species contamination in 603 genome sequencing projects. PeerJ 2:e675.

604 Molyneaux PL, Mallia P, Cox MJ, Footitt J, Willis-Owen SA, Homola D, Trujillo-Torralbo M-B,

605

606

607

608

609

610

611

612

613

614

615

616

617

618

619

620

621

622

623

624

625

626

627

628

629

630

631

632

633

634

635

636

637

638

639

640

641

642

643

644

645

646

647 Elkin S, Kon OM, and Cookson WO. 2013. Outgrowth of the bacterial airway microbiome after rhinovirus exacerbation of chronic obstructive pulmonary disease. American journal of respiratory and critical care medicine 188:1224-1231.

Morgan XC, Kabakchiev B, Waldron L, Tyler AD, Tickle TL, Milgrom R, Stempak JM, Gevers D, Xavier RJ, and Silverberg MS. 2015. Associations between host gene expression, the mucosal microbiome, and clinical outcome in the pelvic pouch of patients with inflammatory bowel disease. Genome Biol 16:637.

Morgan XC, Tickle TL, Sokol H, Gevers D, Devaney KL, Ward DV, Reyes JA, Shah SA, LeLeiko $\mathrm{N}$, and Snapper SB. 2012. Dysfunction of the intestinal microbiome in inflammatory bowel disease and treatment. Genome Biol 13:R79.

Morris A, Beck JM, Schloss PD, Campbell TB, Crothers K, Curtis JL, Flores SC, Fontenot AP, Ghedin E, and Huang L. 2013. Comparison of the respiratory microbiome in healthy nonsmokers and smokers. American journal of respiratory and critical care medicine 187:1067-1075.

Nakayama J, Kobayashi T, Tanaka S, Korenori Y, Tateyama A, Sakamoto N, Kiyohara C, Shirakawa T, and Sonomoto K. 2011. Aberrant structures of fecal bacterial community in allergic infants profiled by $16 \mathrm{~S}$ rRNA gene pyrosequencing. FEMS Immunology \& Medical Microbiology 63:397-406.

Parks DH, Tyson GW, Hugenholtz P, and Beiko RG. 2014. STAMP: statistical analysis of taxonomic and functional profiles. Bioinformatics 30:3123-3124.

Pérez-Losada M, Castro-Nallar E, Bendall M, Freishtat RJ, and Crandall KA. 2015. Dual transcriptomic profiling of host and microbiota during health and disease in pediatric asthma. PloS one Accepted.

Pflughoeft KJ, and Versalovic J. 2012. Human microbiome in health and disease. Annual Review of Pathology: Mechanisms of Disease 7:99-122.

Reuter G. 2001. The Lactobacillus and Bifidobacterium microflora of the human intestine: composition and succession. Current issues in intestinal microbiology 2:43-53.

Round JL, and Mazmanian SK. 2009. The gut microbiota shapes intestinal immune responses during health and disease. Nature Reviews Immunology 9:313-323.

Salter SJ, Cox MJ, Turek EM, Calus ST, Cookson WO, Moffatt MF, Turner P, Parkhill J, Loman NJ, and Walker AW. 2014. Reagent and laboratory contamination can critically impact sequence-based microbiome analyses. BMC biology 12:87.

Scher JU, and Abramson SB. 2011. The microbiome and rheumatoid arthritis. Nature Reviews Rheumatology 7:569-578.

Schizophrenia Working Group of the Psychiatric Genomics Consortium. 2014. Biological insights from 108 schizophrenia-associated genetic loci. Nature 511:421-427.

Sczesnak A, Segata N, Qin X, Gevers D, Petrosino JF, Huttenhower C, Littman DR, and Ivanov II. 2011. The genome of th17 cell-inducing segmented filamentous bacteria reveals extensive auxotrophy and adaptations to the intestinal environment. Cell host \& microbe 10:260-272.

Sebti A, Kiehn TE, Perlin D, Chaturvedi V, Wong M, Doney A, Park S, and Sepkowitz KA. 2001. Candida dubliniensis at a cancer center. Clinical infectious diseases 32:10341038. 
648

649

650

651

652

653

654

655

656

657

658

659

660

661

662

663

664

665

666

667

668

669

670

671

672

673

674

675

676

677

678

679

680

681

682

683

684

685

686

687

688

689

690

691

692

693

Seekatz AM, Aas J, Gessert CE, Rubin TA, Saman DM, Bakken JS, and Young VB. 2014. Recovery of the gut microbiome following fecal microbiota transplantation. MBio 5:e00893-00814.

Segata N, Haake SK, Mannon P, Lemon KP, Waldron L, Gevers D, Huttenhower C, and Izard J. 2012. Composition of the adult digestive tract bacterial microbiome based on seven mouth surfaces, tonsils, throat and stool samples. Genome Biol 13:R42.

Segata N, Izard J, Waldron L, Gevers D, Miropolsky L, Garrett WS, and Huttenhower C. 2011. Metagenomic biomarker discovery and explanation. Genome Biol 12:R60.

Selle K, and Klaenhammer TR. 2013. Genomic and phenotypic evidence for probiotic influences of Lactobacillus gasseri on human health. FEMS microbiology reviews 37:915-935.

Severance EG, Gressitt KL, Stallings CR, Origoni AE, Khushalani S, Leweke FM, Dickerson FB, and Yolken RH. 2013. Discordant patterns of bacterial translocation markers and implications for innate immune imbalances in schizophrenia. Schizophrenia research 148:130-137.

Severance EG, Yolken RH, and Eaton WW. 2014. Autoimmune diseases, gastrointestinal disorders and the microbiome in schizophrenia: more than a gut feeling. Schizophrenia research 14:319-313.

Shoemark DK, and Allen SJ. 2014. The Microbiome and Disease: Reviewing the Links between the Oral Microbiome, Aging, and Alzheimer's Disease. Journal of Alzheimer's Disease 43:725-738.

Storey JD, Taylor JE, and Siegmund D. 2004. Strong control, conservative point estimation and simultaneous conservative consistency of false discovery rates: a unified approach. Journal of the Royal Statistical Society: Series B (Statistical Methodology) 66:187-205.

Strong MJ, Xu G, Morici L, Bon-Durant SS, Baddoo M, Lin Z, Fewell C, Taylor CM, and Flemington EK. 2014. Microbial contamination in Next Generation Sequencing: implications for sequence-based analysis of clinical samples. PLoS pathogens 10:e1004437.

Suez J, Korem T, Zeevi D, Zilberman-Schapira G, Thaiss CA, Maza O, Israeli D, Zmora N, Gilad S, and Weinberger A. 2014. Artificial sweeteners induce glucose intolerance by altering the gut microbiota. Nature 514:181-186.

Tienari P, Sorri A, Lahti I, Naarala M, Wahlberg K, Rönkkö T, Pohjola J, and Moring J. 1985. The Finnish adoptive family study of schizophrenia. The Yale journal of biology and medicine 58:227.

Turnbaugh PJ, Ley RE, Mahowald MA, Magrini V, Mardis ER, and Gordon JI. 2006. An obesity-associated gut microbiome with increased capacity for energy harvest. Nature 444:1027-1131.

van Os J, Kenis G, and Rutten BP. 2010. The environment and schizophrenia. Nature 468:203-212.

Voss M, Wonnenberg B, Honecker A, Kamyschnikow A, Herr C, Bischoff M, Tschernig T, Bals $\mathrm{R}$, and Beisswenger C. 2015. Cigarette smoke-promoted acquisition of bacterial pathogens in the upper respiratory tract leads to enhanced inflammation in mice. Respiratory research 16:41.

Weinstock GM. 2012. Genomic approaches to studying the human microbiota. Nature 489:250-256. 
694 Weiss S, Amir A, Hyde ER, Metcalf JL, Song SJ, and Knight R. 2014. Tracking down the

695

696

697

698

699

700

701

702

703

704

705

706

707

708

709

710

711

712

713

714

715

716

717

718 sources of experimental contamination in microbiome studies. Genome biology 15:564.

White JR, Nagarajan N, and Pop M. 2009. Statistical methods for detecting differentially abundant features in clinical metagenomic samples. PLoS computational biology 5:e1000352.

Wickham H. 2009. ggplot2: elegant graphics for data analysis: Springer Publishing Company, Incorporated.

Wu H-J, Ivanov II, Darce J, Hattori K, Shima T, Umesaki Y, Littman DR, Benoist C, and Mathis D. 2010. Gut-residing segmented filamentous bacteria drive autoimmune arthritis via T helper 17 cells. Immunity 32:815-827.

Ye Y, and Doak TG. 2009. A parsimony approach to biological pathway reconstruction/inference for genomes and metagenomes. PLoS computational biology 5:e1000465.

Yokote H, Miyake S, Croxford JL, Oki S, Mizusawa H, and Yamamura T. 2008. NKT celldependent amelioration of a mouse model of multiple sclerosis by altering gut flora. The American journal of pathology 173:1714-1723.

Yolken R, and Dickerson F. 2014. The microbiome: The missing link in the pathogenesis of schizophrenia. Neurology, Psychiatry and Brain Research 20:26-27.

Yolken RH, Severance EG, Sabunciyan S, Gressitt KL, Chen O, Stallings C, Origoni A, Katsafanas E, Schweinfurth LA, and Savage CL. 2015. Metagenomic sequencing indicates that the oropharyngeal phageome of individuals with schizophrenia differs from that of controls. Schizophrenia bulletin:sbu197. 
Figure $\mathbf{1}_{\text {(on next page) }}$

Oropharyngeal microbial composition at phylum and species levels exhibits different patterns for schizophrenia and control samples

The stacked bar chart shows the most prevalent species present in schizophrenia and controls color-coded by phylum. Green $=$ Actinobacteria; Orange $=$ Bacteroidetes; Blue $=$ Firmicutes; Green = Proteobacteria. The symbol (*) indicates samples from smoker individuals 


\section{OTUs}

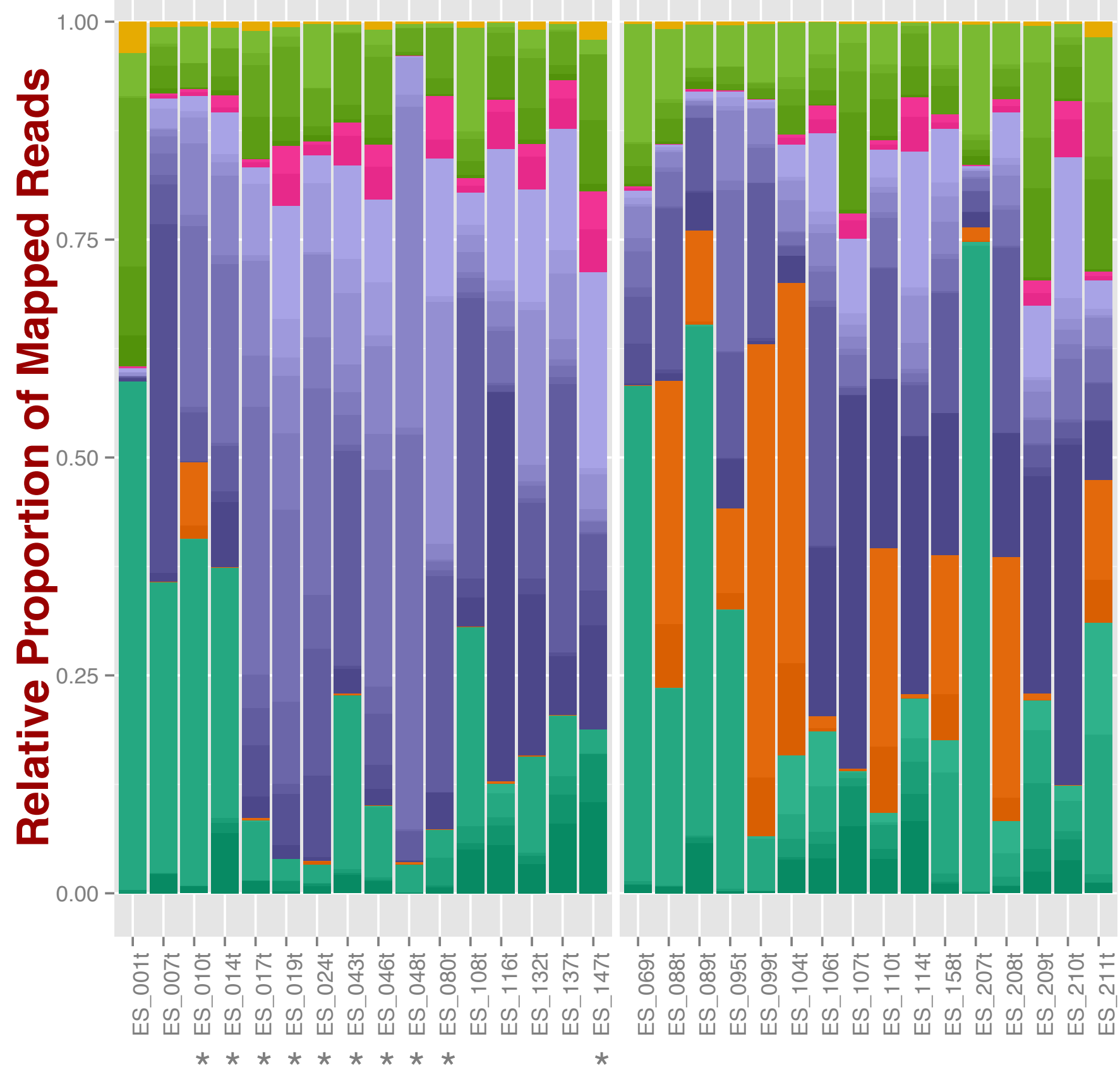

Actinomyces odontolyticus Atopobium parvulum Rothia dentocariosa Rothia mucilaginosa Actinobacteria (other) Prevotella melaninogenica Bacteroidetes (other) Streptococcus gordonii Streptococcus mitis Streptococcus oralis Streptococcus pneumoniae Streptococcus salivarius Streptococcus sp. oral taxon 071 Veillonella parvula Veillonella sp. 3_1_44 Veillonella sp. 6_1_27 Firmicutes (other) Fusobacterium periodonticum Fusobacteria (other) Campylobacter concisus Delftia acidovorans Neisseria flavescens Neisseria subflava Proteobacteria (other) rare (other)

\section{Samples 2015}


Figure 2 (on next page)

Covariation of community structure shows that diversity patterns of samples correlate with disease status, i.e., schizophrenia and controls, and potentially with smoking (at the genus level)

Points represent principal coordinate analysis (PCoA loadings) on Jensen-Shannon Diversity distances. Principal coordinates 1 and 2 in panel A (65\% of variance) and principal coordinates 1 and 3 in panel B (63\% of variance). 


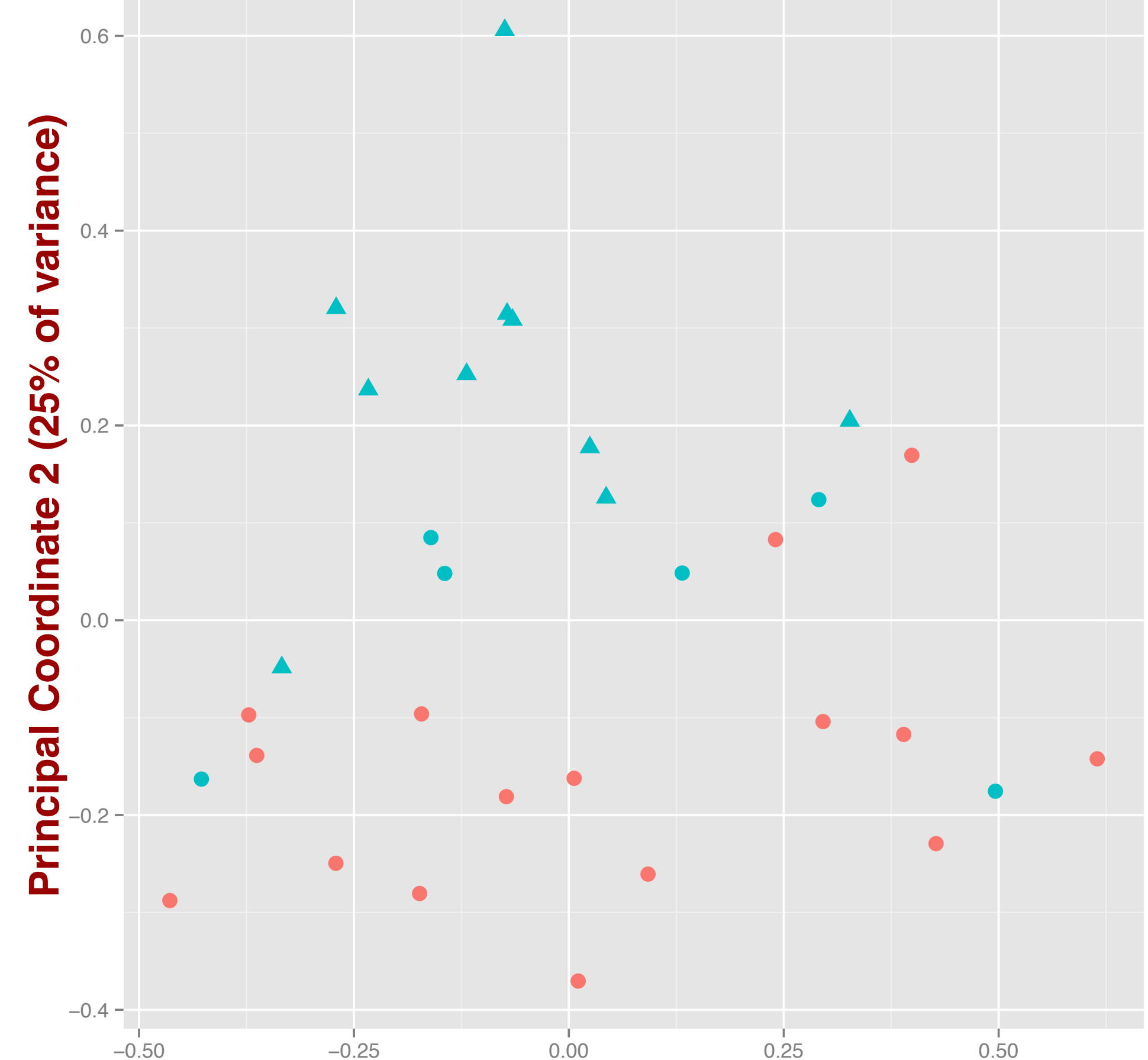

Principal Coordinate 1 ( $40 \%$ of variance)

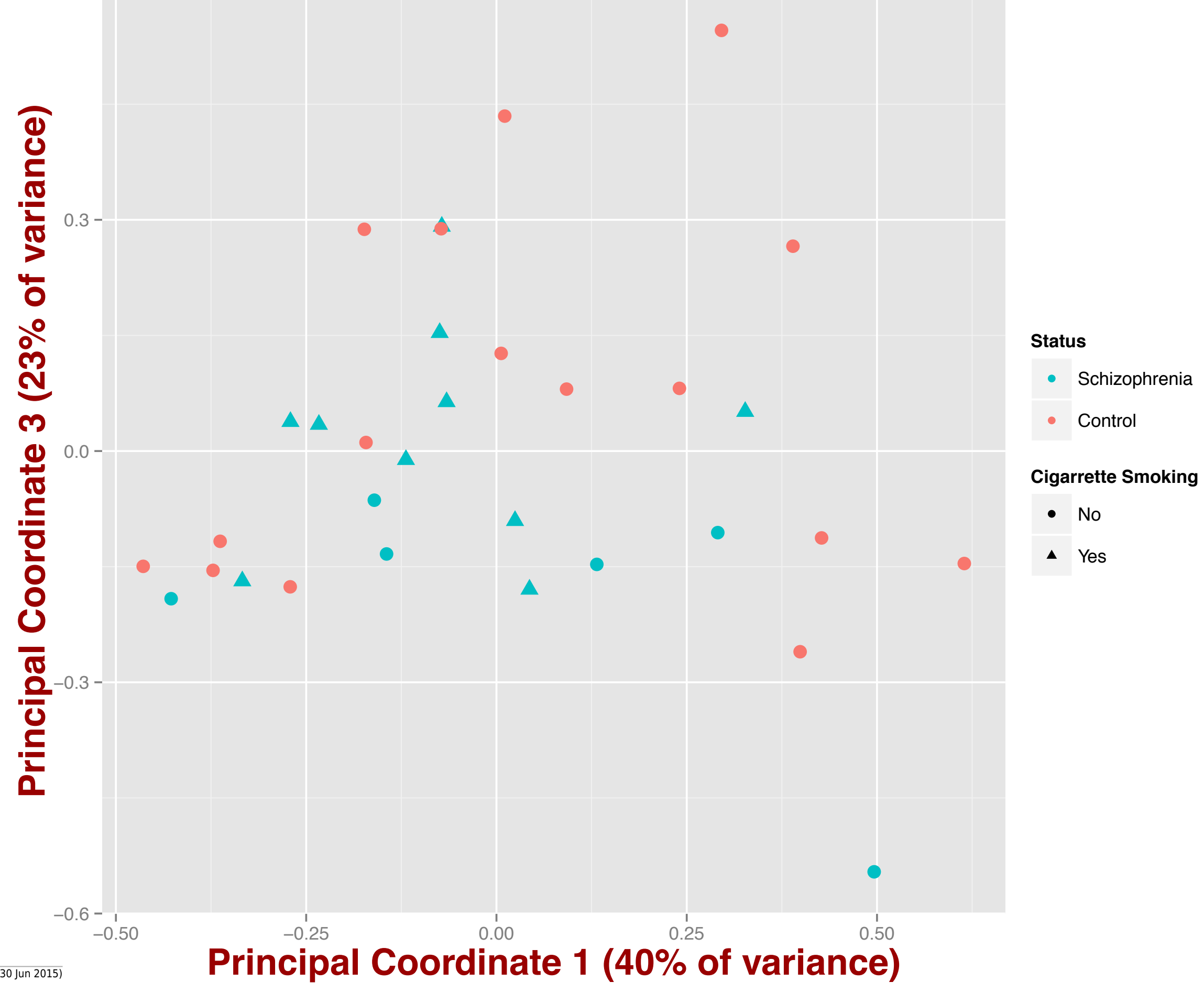


Figure 3 (on next page)

Microbial metabolic pathways with significantly altered abundances in the schizophrenia oropharyngeal microbiome

MXXXXX codes correspond to KEGG modules, i.e., a collection of manually defined functional units (genes). LDA = linear discriminant analysis 
Pyrimidine metabolism: M00051 Cofactor and vitamin biosynthesis: M00115 ATP synthesis: M00144 Central carbohydrate metabolism: M00011 -

Lipopolysaccharide metabolism: M00060 Central carbohydrate metabolism: M00009 Cofactor and vitamin biosynthesis: M00125 Mineral and organic ion transport system: M00300 Cofactor and vitamin biosynthesis: M00123 Central carbohydrate metabolism: M00007 ATP synthesis: M00150 ATP synthase: M00164 ATP synthesis: M00157

Peptide and nickel transport system: M00324 Methane metabolism: M00174 -

Saccharide, polyol, and lipid transport system: M00200 Metallic cation, iron-siderophore and vitamin B12 transport system: M00246

Aromatic amino acid metabolism: M00025

Arginine and proline metabolism: M00028 Phosphate and amino acid transport system: M00233 -

Cysteine and methionine metabolism: M00017 Phosphate and amino acid transport system: M00222 Pyrimidine metabolism: M00053 Serine and threonine metabolism: M00018

Arginine and proline metabolism: M00029 Phosphate and amino acid transport system: M00237 -

Peptide and nickel transport system: M00239 Phosphotransferase system (PTS): M00276 Saccharide, polyol, and lipid transport system: M00194 Saccharide, polyol, and lipid transport system: M00197Purine metabolism: M00049 Purine metabolism: M00050

Samples Control Schizophrenia 


\section{Table $\mathbf{1}$ (on next page)}

Study samples' demographics data

Cases and controls were matched and not statistically different with the exception of smoking condition and body mass index 


\begin{tabular}{|l|l|l|l|l|}
\hline & $\begin{array}{l}\text { Entire Sample } \\
\mathbf{( N = 3 2 )}\end{array}$ & $\begin{array}{c}\text { Controls } \\
\mathbf{( N = 1 6 )}\end{array}$ & $\begin{array}{c}\text { Schizophrenia } \\
\text { cases (non- } \\
\text { smoking; N=6) }\end{array}$ & $\begin{array}{l}\text { Schizophrenia } \\
\text { Cases (all; } \\
\text { N=16) }\end{array}$ \\
\hline Age & $34.5 \pm 7.8$ & $34.3 \pm 10.1$ & $35.9 \pm 3.4$ & $34.7 \pm 4.8$ \\
\hline Male gender & $18 / 32(56.3 \%)$ & $9 / 16(56.3 \%)$ & $3 / 6(50 \%)$ & $9 / 16(56.3 \%)$ \\
\hline White race & $12 / 20(37.5 \%)$ & $5 / 16(31.3 \%)$ & $4 / 6(66.6 \%)$ & $7 / 16(43.8 \%)$ \\
\hline $\begin{array}{l}\text { Mother's } \\
\text { education }\end{array}$ & $13.6 \pm 2.9$ & $14.1 \pm 3.0$ & $14 \pm 3.4$ & $13.1 \pm 2.97$ \\
\hline $\begin{array}{l}\text { Cigarette } \\
\text { smoker }\end{array}$ & $10 / 32(31.3 \%)$ & $0 / 16(0 \%)$ & $0 / 0(0 \%)$ & $10 / 16(62.5 \%)$ \\
\hline $\begin{array}{l}\text { Body Mass } \\
\text { Index }\end{array}$ & $30.1 \pm 7.0$ & $25.5 \pm 4.5$ & $33.4 \pm 7.5$ & $34.7 \pm 6.0$ \\
\hline
\end{tabular}




\section{Table 2 (on next page)}

Microbial species relatively more abundant in schizophrenia samples than in controls

Effect size represents the size of the difference of schizophrenia samples over controls. The effect size as an associated standard error, and multiple comparisons were adjusted using the Benjamini-Hochberg procedure (BH). Asterisk indicates that Eubacterium hallii has been associated with smoking in the nasopharynx microbiome (PMID: 21188149) 


\begin{tabular}{|c|c|c|c|c|c|c|}
\hline $\begin{array}{c}\text { Effect } \\
\text { Size } \\
\text { (log2 } \\
\text { Fold } \\
\text { Change) }\end{array}$ & $\begin{array}{l}\text { Effect } \\
\text { Size } \\
\text { Standard } \\
\text { Error }\end{array}$ & $\begin{array}{c}p \text { value } \\
\text { (BH } \\
\text { adjusted) }\end{array}$ & Phylum & Genus & Species & Description \\
\hline 8.37 & 1.17 & $2.55 \mathrm{E}-10$ & Firmicutes & Lactobacillus & Lactobacillus gasseri & $\begin{array}{l}\text { Lactic acid bacterium. } \\
\text { Member of diverse } \\
\text { communities including } \\
\text { gut, vaginal, and oral } \\
\text { microbiome. Appears to } \\
\text { be the main species of } \\
\text { lactobacilli that inhabits } \\
\text { the human } \\
\text { gastrointestinal tract }\end{array}$ \\
\hline 6.81 & 0.99 & $9.61 \mathrm{E}-10$ & Firmicutes & Catenibacterium & Catenibacterium mitsuokai & $\begin{array}{l}\text { Phylogenetic relative of } \\
\text { lactobacilli. Found in } \\
\text { gastrointestinal tract }\end{array}$ \\
\hline 4.82 & 0.99 & $3.94 \mathrm{E}-05$ & Firmicutes & Eubacterium & Eubacterium hallii* & $\begin{array}{l}\text { Butyrate forming, } \\
\text { Lactate-utilizing } \\
\text { bacterium. Present in } \\
\text { human feces }\end{array}$ \\
\hline 5.71 & 1.29 & 3.13E-04 & Ascomycota & Candida & Candida dubliniensis & $\begin{array}{l}\text { Opportunistic fungus. } \\
\text { Part of the oral fungal } \\
\text { microbiome. Present in } \\
\text { periodontal disease }\end{array}$ \\
\hline 2.98 & 0.80 & 4.17E-03 & Firmicutes & Lactobacillus & Lactobacillus salivarius & $\begin{array}{l}\text { Lactic acid bacterium. } \\
\text { Member of diverse } \\
\text { communities including } \\
\text { vaginal and oral } \\
\text { microbiome }\end{array}$ \\
\hline 3.79 & 1.06 & $6.30 \mathrm{E}-03$ & $\begin{array}{c}\text { Actinobacte } \\
\text { ria }\end{array}$ & Bifidobacterium & $\begin{array}{c}\text { Bifidobacterium } \\
\text { pseudocatenulatum }\end{array}$ & $\begin{array}{l}\text { Lactic acid bacterium. } \\
\text { Gastrointestinal tract, } \\
\text { vagina and mouth of } \\
\text { mammals, including } \\
\text { humans }\end{array}$ \\
\hline
\end{tabular}

1 